\title{
DISTRIBUCIÓN DE LA RIQUEZA, COMPOSICIÓN TAXONÓMICA Y GRUPOS FUNCIONALES DE HORMIGAS DEL SUELO A LO LARGO DE UN GRADIENTE ALTITUDINAL EN EL REFUGIO DE VIDA SILVESTRE LAQUIPAMPA, LAMBAYEQUE- PERÚ
}

\author{
DISTRIBUTION OF THE RICHNESS, TAXONOMIC COMPOSITION AND \\ FUNCTONAL GROUP OF SOIL'S ANTS ALONG AN ALTITUDINAL GRADIENT \\ AT LAQUIPAMPA WILDLIFE REFUGE, LAMBAYEQUE - PERU
}

\author{
Silvia Castro Delgado ${ }^{1}$, Clorinda Vergara Cobian ${ }^{1}$, Consuelo Arellano Ugarte ${ }^{2}$
}

\begin{abstract}
Resumen
En este estudio, se evaluó la distribución de la riqueza, composición taxonómica y grupos funcionales de hormigas del suelo a lo largo de un gradiente altitudinal en Laquipampa (Lambayeque - Perú). Tres estratos altitudinales fueron establecidos en el área de estudio: alto $(1824-2023 \mathrm{msnm})$, medio $(1362-1433 \mathrm{msnm})$ y bajo $(600-810 \mathrm{msnm})$. En cada estrato, se ubicaron dos montañas y en cada una, se establecieron dos estaciones de muestreo, una en la cima y la otra, al piedemonte. En cada estrato hubo cuatro estaciones. En total, se registraron 892 individuos y 34 morfoespecies, todos colectados en 96 trampas pitfall. La colecta manual registró 30 morfoespecies. La combinación de ambos métodos resultó en un total de 49 morfoespecies pertenecientes a 21 géneros y 6 subfamilias. En general, se estableció que a nivel regional, la riqueza disminuyó de manera lineal con el aumento de la altitud. Asimismo, se determinó que a nivel local la riqueza fue menor en la cima que en el piedemonte. La temperatura y la humedad relativa se relacionan a la fluctuación de la riqueza tanto a nivel local como a nivel regional, ya que la temperatura disminuyó y la humedad aumentó, ambas de forma lineal, con el incremento de la altitud. Todos los estratos fueron dominados por omnívoros. La composición de morfoespecies fue muy similar entre las estaciones cercanas, sin embargo, más del $50 \%$ de morfoespecies registradas en cada estrato podrían ser particularmente propias, lo que sugiere la especial importancia de cada tipo de bosque.
\end{abstract}

Palabras clave: Hormigas, Riqueza, Gradiente altitudinal, Escala

\begin{abstract}
We surveyed species richness, taxonomic composition and functional groups for soil ants along an altitudinal gradient at Laquipampa (Lambayeque - Peru). We selected three strata in the area: high (1824 - 2023 meters high), middle (1362 - 1433 meters high) and low (600 - 810 meters high). In each stratum we located two mountains, and for each mountain we selected two sampling sites, one at the mountaintop and one at the foothill. Thus, in each stratum there were four sampling sites (stations), with a total of 12 sampling stations, 6 at the mountaintops and 6 at the foothills. A total of 892 individuals and 34 species were registered in 96 pitfall traps. Manual collection found 30 species. The combination of both methods resulted in 49 species belonging to 21 genus and 6 subfamilies. At the regional level, the number of species decreased linearly as altitude increased. Overall, the number of species was smaller at the top of the mountain than at its foothill. Temperature, relative humidity and their combined effect can be used to explain the fluctuation in the number of species both locally and regionally, since temperature diminished and humidity increased with altitude. All strata were dominated by omnivorous ants. More than $50 \%$ of the species, in each stratum were not found in other strata, suggesting the importance of each kind of forest.
\end{abstract}

Key words: Ants, Richness, Altitudinal gradient, Scale

\section{Introducción}

La región de los Andes del Norte ha sido nombrada una de las 237 regiones biogeográficas más importantes del mundo debido a su alta diversidad biológica y alto endemismo (Dinerstein et al., 1995).
No obstante, gran parte de esta diversidad e integridad, se pierde por el acelerado incremento de las perturbaciones antropogénicas negativas (Kattan, 2002; Krincher, 2006). Numerosos casos han sido reportados (Dourojeanni, 2006); en la década de 1981 
a 1990, América Tropical perdió 16.6 millones de hectáreas de bosques de montaña (Whitmore, 1997 citado por Kattan, 2002). La importancia de conservar y manejar de forma adaptativa los bosques de montañas se debe a que estos desempeñan muchas funciones elementales en los ecosistemas ecológicos sociales, como captar agua, evitar la erosión de los suelos, su empobrecimiento y controlar los desprendimientos de tierras debido a las elevadas pendientes y a las lluvias (Dudley \& Stolton, 2003). Asimismo, las regiones montañosas hospedan un gran número de especies de los cuales un gran porcentaje son endémicas. En general, las dimensiones verticales de las montañas generan gradientes de temperaturas, precipitación e insolación que pueden determinar la variación en la distribución y la abundancia de las especies dando lugar de este modo a una gran variedad de "zonas de vida" (Holdrige, 1982).

El Refugio de Vida Silvestre Laquipampa (Ferreñafe, Lambayeque), antiguamente Zona Reservada, fue establecida para la preservación, conservación e investigación científica de la pava aliblanca y otras especies de flora y de fauna. Se encuentra en la vertiente occidental andina del norte del Perú y presenta una topografía montañosa que forma un gradiente altitudinal cuyas cumbres más bajas (400 msnm) se encuentran al sudoeste y las más altas (como el cerro "La Punta" $2600 \mathrm{msnm}$ ) al noreste (Flanagan \& Angulo, 2003). Esta zona montañosa cuyas alturas disminuyen de norte a sur representa una oportunidad para estudiar y entender patrones de diversidad a través de gradientes altitudinales tanto a escalas locales, en una montaña, como a escalas regionales (regiones montañosas).

Las tendencias altitudinales observadas en la riqueza de especies varían entre grupos de organismos y de área a área. Sin embargo, la mayoría de los estudios de diversidad en gradientes altitudinales con varios taxa han resultado en dos patrones: disminución de la riqueza de especies con el incremento de la altura, y picos máximos de riqueza a alturas medias, el más ampliamente aceptado (Terborgh, 1977; Rahbek, 1995, Grytnes \& McCain, 2007). Factores climáticos, biológicos, ecológicos, geográficos, históricos y estocásticos han sido sugeridos como las causas de la variación altitudinal de la riqueza de especies (Rahbek, 1995; Sanders et al., 2003; Sanders et al., 2007; Grytnes \& McCain, 2007).

Los insectos han sido ampliamente utilizados en estudios de patrones de diversidad temporal y espacial (Giraldo, 2002; Giraldo \& Arellano, 2003). Por su sensibilidad a las perturbaciones antropogénicas, los han propuesto para monitorear ecosistemas (Rosenberg et al., 1986) y por su alta diversidad y abundancia, para ayudar a identificar zonas prioritarias para su conservación (Kremen et al., 1993; Pearson \& Cassola, 1992).
Las hormigas están entre los grupos de insectos más ampliamente estudiados (www.antbase.org (2008)). Entre las características que las hacen componentes importantes de la biodiversidad están 1) $\mathrm{Su}$ alta abundancia, pues constituyen una enorme proporción de la biomasa animal terrestre (Fittkau \& Klinge, 1973; Borror et al., 1992; Davidson \& PatrellKim, 1996; Davidson, 1998; Davidson et al., 2003; ) y b) $\mathrm{Su}$ alta riqueza de especies (12463 especies registradas hasta la fecha según www.antbase.org (2008)), cuyos mayores registros han sido reportados para el Neotrópico y particularmente, para Perú (Wilson, 1987; Verhaagh, 1990; 1991). Asimismo, tienen una amplia distribución en muchas áreas biogeográficas y cubren una amplia variedad de hábitats en la tierra (Wheeler, 1965; Holldobler \& Wilson, 1990; Borror et al., 1992; Bolton, 1994; 1995). Las hormigas ocupan un amplio rango de nichos ecológicos que en muchos casos son importantes para el funcionamiento de los ecosistemas (Holldobler \& Wilson, 1990; Alonso \& Agosti, 2000; Folgarait, 1998). Un grupo importante es aquel que forrajea $\mathrm{y}$ anida en el suelo ya que pueden enriquecerlo al retornar los nutrientes (Haines, 1978; Gunadi \& Verhoef, 1993). En lugares donde las hormigas llegan a densidades elevadas, pueden mover la misma cantidad de suelo que las lombrices de tierra. Debido a que transportan restos de animales y plantas dentro de sus nidos bajo el suelo, mezclan estos materiales con la tierra escavada y el área del nido es cargada con altos niveles de carbono, nitrógeno y fósforo. Consecuentemente, el suelo se fragmenta en un mosaico de concentración de nutrientes (Brise, 1982; Holldobler \& Wilson, 1990). Algunos investigadores en Australia han utilizando a las hormigas como bioindicadores del grado de disturbio y de la restauración de ecosistemas (Majer, 1983; Andersen, 1991; 1993; 2000; Andersen et al., 2003; Andersen \& Majer, 2004).

Estudios de diversidad de hormigas en gradientes altitudinales también han reportado disminución de la riqueza de especies con la altitud (Brühl et al., 1999) y picos máximos de riqueza a altitudes medias (Olson, 1994; Samson et al., 1997; Sanders, 2002). Aunque asimismo, se ha encontrado casos particulares donde la riqueza de especies aumenta con la altitud (Sanders et al., 2003).

En el Perú, muy pocos estudios con comunidades de insectos en gradientes altitudinales han sido realizados y ninguno con comunidades de hormigas. Dada la importancia de este grupo, este estudio espera entender los patrones de distribución de la riqueza, de la composición taxonómica, de los grupos funcionales así como el cambio de la composición de especies a lo largo de la gradiente altitudinal a nivel local (en una montaña) y regional (en una gradiente altitudinal de habitats o estratos) en el refugio de vida silvestre Laquipampa. Se hizo además, una evaluación de 
variables abióticas como la temperatura y la humedad relativa para explicar el cambio de la riqueza de hormigas con la altitud a nivel regional y local.

\section{Materiales y métodos}

$\underline{\text { Área de estudio }}$

El refugio de Vida Silvestre Laquipampa, fue creado inicialmente como Zona Reservada en 1982, y posteriormente como refugio, el 11 de Julio del 2006. Esta área esta situada en el Distrito de Incahuasi, Provincia de Ferreñafe (Departamento de Lambayeque, Perú - Figura 1), abarca 11.346 has e incluye altitudes que van desde los $400 \mathrm{~m}$ al sudoeste (70 33' 40' 'W, 6 24' 20' 'S) hasta el cerro 'La Punta' $\left(79^{\circ} 28^{\prime} \mathrm{W}, 6^{\circ} 15^{\prime} 45^{\prime \prime} \mathrm{S}\right)$ de $2600 \mathrm{~m}$, en el norte. Los límites este y sur están a altitudes bajas, coincidiendo aproximadamente con el curso del Río del La Leche. Los límites norte y oeste coinciden con las crestas más altas, las cuales disminuyen en altitud de norte a sur. Normalmente, los meses de lluvias se extienden desde Diciembre hasta Marzo (Flanangan \& Angulo, 2003).

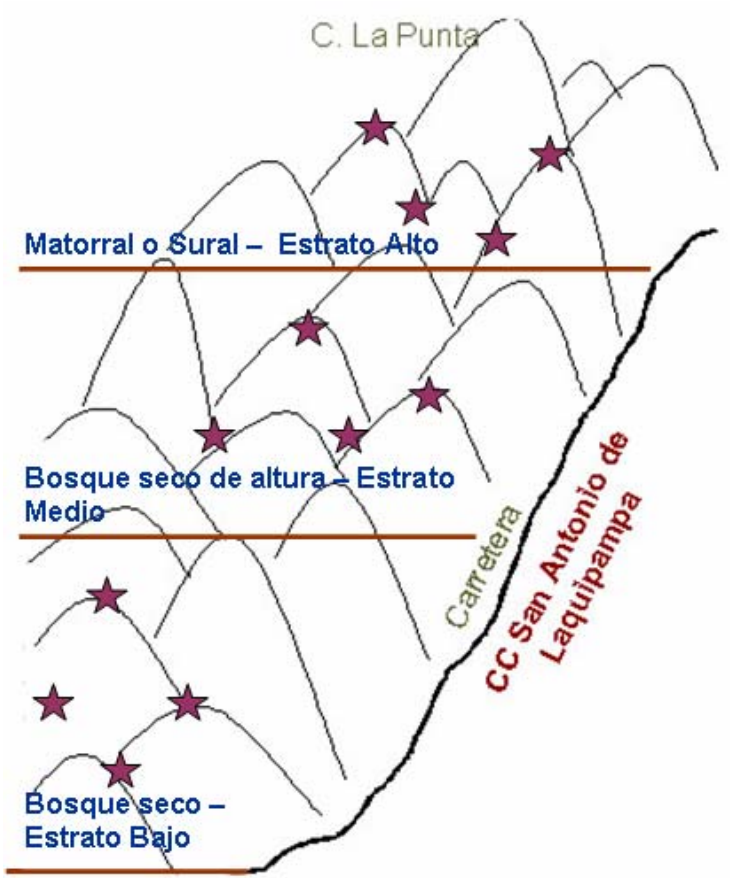

Figura 1. Distribución de las estaciones de muestreo en los tres estratos altitudinales en Laquipampa.

Laquipampa es un gradiente altitudinal de montañas que ha dado como resultado tres principales tipos de hábitats (Angulo \& Alemán de Lama, 2006): 1) "Bosque Seco", cuyo rango altitudinal va desde los 240 hasta los 1300 a 1400 metros de altitud, ocupa gran parte del refugio y esta conformado por bosques semi-deciduos. Las especies vegetales predominantes son: el hualtaco (Loxopterygium huasango), el palo blanco (Celtis iguanea), el palo santo (Bursera graveolens), el pasallo (Eriotheca ruizii), el higuerón
(Ficus padifolia), el huayrul (Erythrina smithiana), el overo (Cordia lutea) y el chaquirón (Pithecellobium excelsum). El muestreo de este bosque se realizó en la quebrada Negrahuasi-Piedra Loza (UTM: 667575 9297878 - en este estudio fue denominado Estrato Bajo); 2) El "Bosque seco de altura", tiene como plantas predominantes al cedro de altura (Cedrela montana), el higuerón (Ficus padifolia), el huayrul (Erythrina smithiana), el shuco (especie indeterminada) y el charán o paipai (Caesalpinea paipai). Estos bosques se encuentran por encima de los 1400 hasta los $1800 \mathrm{msnm}$ aproximadamente y se caracterizan porque la vegetación es perenne. Este hábitat es en general mucho más húmedo que el anterior, y esto se refleja en la gran cantidad de orquídeas, culantrillos, helechos y líquenes que se encuentran dentro de él. En este estudio, este tipo de bosque fue muestreado en las partes altas de la quebrada Shambo - La Botija (UTM: 669861 9303166 - Estrato Medio) y 3) El "Matorral - Sural" que se encuentra por encima de los 1800 hasta los $2500 \mathrm{msnm}$, tiene como especies vegetales predominantes al suro (Chusquea sp.), el cedro de altura (Cedrela montana), la taya o tara (Caesalpinea spinosa) y el chachacomo (especie indeterminada), una gran variedad de arbustos, líquenes y musgo. Se caracteriza porque la vegetación contiene muy pocos árboles (excepto en las quebradas), el terreno esta casi completamente cubierto de matorrales y de suro, la mayoría cubierto de musgo. Las temperaturas son bajas en época húmeda y la humedad puede llegar al $100 \%$ por la presencia de las neblinas. El muestreo de este hábitat se realizó en la quebrada Salsipuedes Corral Grande (UTM: 671089 - 9305408 - Estrato Alto).

Estaciones de muestreo para medir el efecto altitudinal sobre la riqueza de especies de hormigas

El muestreo fue realizado en 12 estaciones de muestreo que fueron distribuidos a nivel regional (en un rango de montañas), en tres estratos altitudinales: Estrato Bajo (B), Estrato Medio (M) y Estrato Alto (A), correspondientes a los habitats "Bosque Seco", "Bosque Seco de altura" y "Matorral o Sural", respectivamente. A nivel local (en una montaña), en cada estrato se seleccionaron al azar dos montañas y en cada una se ubicaron 2 estaciones (Figura 1), una al pie de monte, al lado de una quebrada (A) y la otra estación, en la cima de la misma montaña (B). El muestreo en el punto medio altitudinal de la montaña no pudo ser realizado porque en muchos casos las pendientes eran muy empinadas y de difícil acceso. El rango altitudinal de las estaciones de muestreo abarco desde los 600 a los 2023 msnm: B(A)-1: 632; B(B)-1: 757; B(A)-2: 600; B(B)-2: 810; M(A)-1: 1362; M(B)1: 1388; M(A)-2: 1374; M(B)-2: 1433; A(A)-1: 1833; A(B)-1: 2023; A(A)-2: 1824 y A(B)-2: 2014 msnm. Por limitaciones logísticas, el muestreo sólo se realizó durante la época húmeda, desde el 12 - 24 de Febrero 
del 2006 y no se pudo incluir puntos bajo los 600 msnm.

Muestreo de hormigas

En cada una de las 12 estaciones se utilizaron dos técnicas de muestreo: Trampas pitfall y colecta manual. Tanto el análisis del muestreo, como todos los análisis de riqueza $(\mathrm{S})$, diversidad beta, distribución de especies, composición taxonómica y porcentajes de los grupos funcionales fueron calculados sólo con los registros obtenidos de las trampas pitfall. Los resultados de la colecta manual fueron analizados de forma separada. Los registros de ambos muestreos solo se mezclaron para obtener la riqueza "gamma región" (la riqueza total de toda la región evaluada). Trampas pitfall

En cada estación de muestreo se establecieron dos transectos de $80 \mathrm{~m}$ de largo cada uno, paralelos a la pendiente, paralelas entre sí y separados aproximadamente $20 \mathrm{~m}$. En cada transecto se colocaron 4 trampas pitfall, cada una separada de la otra en 20 metros. El esfuerzo temporal de muestreo de todas las trampas fue de 84 horas. En total, en toda la zona, se colocaron 96 trampas pitfall. La trampa consistió de un vaso plástico, de $10 \mathrm{~cm}$ de diámetro por $15 \mathrm{~cm}$ de profundidad, semi lleno con formol diluido al $5 \%$ y 0.5 gr de detergente para romper la tensión superficial del líquido, enterrado al ras del suelo.

Colecta Manual

En cada sitio de muestreo se efectúo una colecta manual de sólo hormigas del suelo, en un transecto de $80 \mathrm{~m} \mathrm{x} 1 \mathrm{~m}$ y por 60 minutos. Estos fueron paralelos y separados por 50 metros de los transectos de las trampas pitfall. Dos estaciones de muestreo de cada estrato fueron muestreados en un sólo día, de 9:00 am a 1:00 pm, así las cuatro estaciones de cada estrato fueron muestreados en dos días y los tres estratos fueron muestreados por una sola persona. Este se realizó con ayuda de pinzas entomológicas, aspirador y un envase plástico con alcohol al $70 \%$ en donde se colocaron las hormigas capturadas en todo el transecto. El resultado final fueron 12 transectos de captura manual para toda la zona.

Laboratorio

Posteriormente, en el laboratorio, el contenido de cada trampa pitfall se tamizó empleando un cernidor muy fino para deshacerse de la arena y piedras. El material biológico, compuesto por artrópodos en general, se conservó en el mismo envase debidamente etiquetado para su posterior conteo e identificación. Al principio, las hormigas fueron separadas, contadas como morfoespecies y montadas en alfileres entomológicos $\mathrm{y} / \mathrm{o}$ trasladadas a frascos de vidrio con alcohol a los 70\%. Posteriormente, fueron identificados hasta género y/o especie utilizando las claves de Bolton (1994) y Fernández (2003). Aquellas a las que no se pudo determinar a la categoría de especie, se identificaron como morfoespecie dejándolo así para su posterior identificación (Derraik et al., 2002). Cada morfoespecie está designada por el nombre del género al que pertenecen, seguido por el número correspondiente (Morfoespecie $1=$ Mor 1). Los especimenes fueron depositados en el Museo de Entomología de la Universidad Nacional Agraria La Molina (Lima, Perú).

Temperatura y Humedad a nivel del suelo

Se evaluó la influencia del clima sobre la riqueza de especies, debido a que los extremos climáticos pueden tener efectos directos sobre la distribución de especies. La temperatura y la humedad se midieron colocando el termohidrómetro al nivel del suelo en 6 puntos al azar de cada transecto durante el tiempo de colocación de las trampas y entre las 9:00 am y 1:00 pm. Para los análisis se utilizaron la temperatura y la humedad promedio de cada estación de muestreo.

Análisis de los datos

Análisis del muestreo: curvas especie - área

Debido a que al cambiar el orden en el proceso de acumulación de muestras se obtendrán curvas de diferentes formas, el análisis de estas curvas se realizó con los parámetros $\mathrm{R}^{2}, \mathrm{~S}_{\mathrm{obs}} /(\mathrm{a} / \mathrm{b}), \mathrm{n}_{0.95} \mathrm{y} \mathrm{n}_{0.70}$, dados por el ajuste a la ecuación de Clench ${ }^{*}$, para valorizar la calidad del muestreo y saber si el esfuerzo del muestreo o el tamaño de la unidad muestral (TUM) tomado durante 84 horas ( 3 y $1 / 2$ dias) fue suficiente para capturar un gran porcentaje de especies. El análisis de estas curvas con estos parámetros sirve además para extrapolar el número de especies observadas y estimar el total de especies que estarían presentes en la zona. Asimismo, se puede planificar el esfuerzo de muestreo que se debe invertir en evaluaciones posteriores y obtener resultados más fiables (Jiménez-Valverde \& Hortal, 2003). En este estudio, el análisis se realizó en 6 estaciones de muestreo elegidas al azar.

\section{Análisis de la riqueza de especies}

La riqueza (S) se analizó bajo un criterio espacial de tres escalas: 1) Escala "alfa", en el cual se consideró el muestreo de cada estación (12 unidades de análisis en total); 2) "Gamma estrato" en donde se consideró como unidad de análisis la agrupación total de las 4 estaciones de muestreo en cada estrato (3 unidades de análisis en total) y 3) "Gamma región" o la riqueza total de toda la región evaluada. Para hallar diferencias significativas $(p<0.05)$ entre la riqueza de especies de los tres estratos y entre la riqueza del piedemonte y la cima de la montaña se realizó un análisis de varianza de dos vías (Steel \& Torrie, 1988), en el cual se tomaron en cuenta dos factores: Estrato (Bajo, Medio y Alto) y Topografía (Cima (B) y Piedemonte (A)). Para este análisis se utilizaron los

$$
\begin{aligned}
* S_{n} & =a . n /(1+b . n), \\
n_{0.95} & =0.95 /[b .(1-0.95)],
\end{aligned}
$$


valores en la escala alfa comprobando previamente su distribución normal mediante la prueba AndersonDarling y probando el supuesto de Homogeneidad de varianzas con la prueba Bartlett. De forma complementaria, se muestran los promedios de las riquezas a través de los estratos con sus respectivos intervalos de confianza al $95 \%$ calculados a partir de la distribución t (Steel \& Torrie, 1988). La relación entre la riqueza de especies (variable dependiente) y la temperatura y humedad (variables independientes) se analizó mediante análisis de correlación y sus correspondientes diagramas de dispersión (Steel \& Torrie, 1988). Para los diversos análisis se utilizaron los programas PRIMER 5.0 (Clarke \& Warwick, 1996) y STATISTICA 7.0 (StatSoft, Inc., 2004). Diversidad beta

El cambio de la composición de especies a lo largo del gradiente altitudinal fue calculado utilizando el índice de Jaccard con los cuales se construyó un dendrograma (Magurran, 1988).

Grupos funcionales

Para el análisis de la distribución altitudinal de los grupos funcionales en la escala "Gamma estrato", se realizó una clasificación de las especies colectadas, para la cual fueron consideradas 4 categorías de acuerdo a los estudios de Silvestre et al. (2003); Andersen et al. (2003); Andersen (2000) y Brown (2000): 1) Cortadoras (especies polimórficas de colonias grandes que cultivan hongos a partir de la colecta de hojas frescas, como las especies de Atta sp.); 2) Omnívoros (dominantes omnívoras del suelo como Pheidole sp., Solenopsis sp., Megalomyrmex sp.; oportunistas del suelo como Brachymyrmex sp.; Camponotinas patrulleras generalistas, como Camponotus sp. y arbóreas pequeñas de reclutamiento masivo como Crematogaster sp.); 3) Depredadoras (depredadoras grandes epigeas, como Odontomachus sp., Pseudomyrmex sp., depredadoras nómadas como Labidus sp.) y 4) Crípticas (attinas crípticas, como Cyphomyrmex sp., que son cultivadoras de hongos sobre material en descomposición). Para calcular la abundancia relativa de cada grupo funcional, la abundancia total de cada especie en cada trampa fue considerada en una escala de 5 puntos: $1=1$ hormiga; $2=2-5$ hormigas; $3=6-20$ hormigas, $4=21-50$ hormigas, $5=>50$ hormigas. Esto se hizo para reducir las serias distorsiones causadas por el problema de encontrar numerosos individuos de una única colonia dentro de una o pocas trampas (Andersen, 1991, 1993, 2000; Andersen et al., 2003)

Distribución de cada especie

Para hallar el rango de la amplitud de la distribución de cada especie a través de la gradiente sólo se tomo en cuenta su presencia en cada trampa y se calculó el porcentaje de trampas donde estuvo presente una determinada especie.

\section{Resultados y discusión}

Análisis del muestreo: curvas especie - área

Las curvas de acumulación de especies se muestran en la Figura 2. Casi todas las curvas muestran cierta tendencia a alcanzar las asíntotas. Los parámetros $\left(\mathrm{R}^{2}, \mathrm{~S}_{\mathrm{obs}} /(\mathrm{a} / \mathrm{b})\right.$, número de especies estimadas, $\mathrm{n}_{0.95}$ y $\mathrm{n}_{0.70}$ ) proporcionados por el ajuste de las curvas a la ecuación de Clench se muestran en la Tabla 1. Todas las curvas presentan coeficientes de determinación $\left(\mathrm{R}^{2}\right)$ cercanos a 1 , lo que indica un buen ajuste al modelo. Los parámetros $\mathrm{S}_{\mathrm{obs}} /(\mathrm{a} / \mathrm{b})$ calculados para las estaciones $\mathrm{A}(\mathrm{A})-1, \mathrm{~A}(\mathrm{~B})-2, \mathrm{M}(\mathrm{A})-1, \mathrm{M}(\mathrm{A})-2$, $\mathrm{B}(\mathrm{A})-1$ y $\mathrm{B}(\mathrm{A})-2$ muestran que con 8 trampas y un esfuerzo temporal de 84 horas (3 y $1 / 2$ días) se registraron entre el $50 \%$ al $64 \%$ del total de especies estimadas. En consecuencia, si se quiere conocer el 95\% de las especies en cada estación de muestreo se deberían utilizar entre 88 y 155 trampas. Debido a que el balance entre los costos (esfuerzo adicional en tiempo y dinero) y las ganancias (número de especies nuevas) se hace desfavorable, muchos investigadores suelen establecer límites arbitrarios (JiménezValverde \& Hortal, 2003). Considerando que sólo se están estudiando las especies del suelo y que probablemente, muchas de las especies capturadas que viven en el dosel pueden ser visitantes ocasionales de éste, en esta ocasión, se podría considerar como límite arbitrario el $70 \%$ de las morfoespecies. Así, con este criterio, para alcanzar este porcentaje, el análisis recomienda emplear entre 11 a 19 trampas. No obstante, probablemente, el número de trampas requerido disminuiría si se incrementa el tiempo de muestreo, es decir, si se dejaran las trampas más de 84 horas. En el caso de las hormigas, muchos investigadores recomiendan utilizar varios tipos de metodologías para el muestreo, como trampas pitfall y Winkler, además de una colecta manual (Alonso \& Agosti, 2000). Aunque en este estudio se realizó una colecta manual se optó por no incluirla en los análisis estadísticos debido a que es una metodología sesgada en el sentido que depende de la hora a la que se este colectado y las condiciones climáticas en esas horas de muestreo.

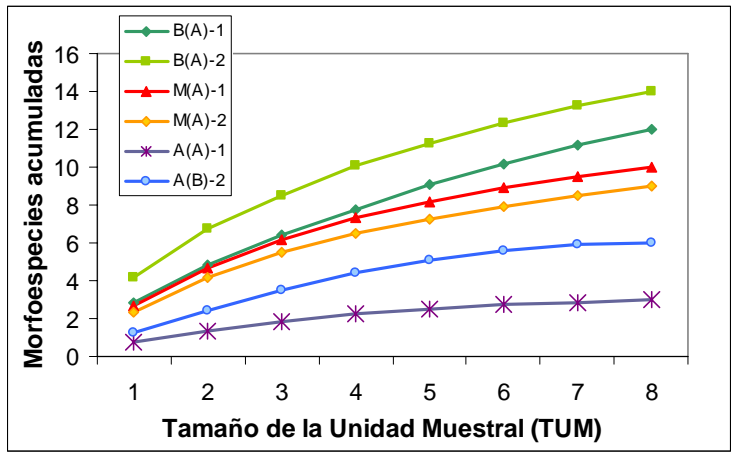

Figura 2. Curvas de acumulación de especies para seis estaciones de muestreo 
Riqueza total de toda la región evaluada

Se ha registrado un total de 892 individuos distribuidos en 34 morfoespecies, todos colectados en 96 trampas pitfall. La colecta manual registró un total de 30 morfoespecies. La combinación de ambos métodos, resultó en un total de 49 morfoespecies de hormigas pertenecientes a 21 géneros y 6 subfamilias (Tabla 2). Los dos métodos de muestreo generaron dos ensamblajes propiamente diferentes ya que sólo tuvieron 15 morfoespecies (30\%) en común.

Riqueza gamma estrato y riqueza alfa

A nivel regional, el calculo de la riqueza en la escala gamma estrato mostró 21, 16 y 10 morfoespecies en los estratos Bajo, Medio y Alto, respectivamente, lo que muestra que esta riqueza disminuye linealmente con la altitud. Similarmente, el análisis de varianza de dos vías utilizando la riqueza alfa promedio muestra que por lo menos la riqueza entre uno de los pares de estratos es diferente $(\mathrm{F}=$ $18.3, \mathrm{gl}=2, \mathrm{P}<0.01)$. La riqueza promedio de cada estrato y sus intervalos de confianza (Figura 3) muestran que no hay diferencias significativas entre la riqueza del estrato bajo y el estrato medio y entre el estrato medio y el alto, lo cual sugiere que el estrato medio es una transición entre los adyacentes. Pero si hay diferencias significativas entre el estrato bajo $\mathrm{y}$ alto $(\mathrm{P}<0.05)$, siendo la riqueza del estrato bajo mucho mayor que la del alto.

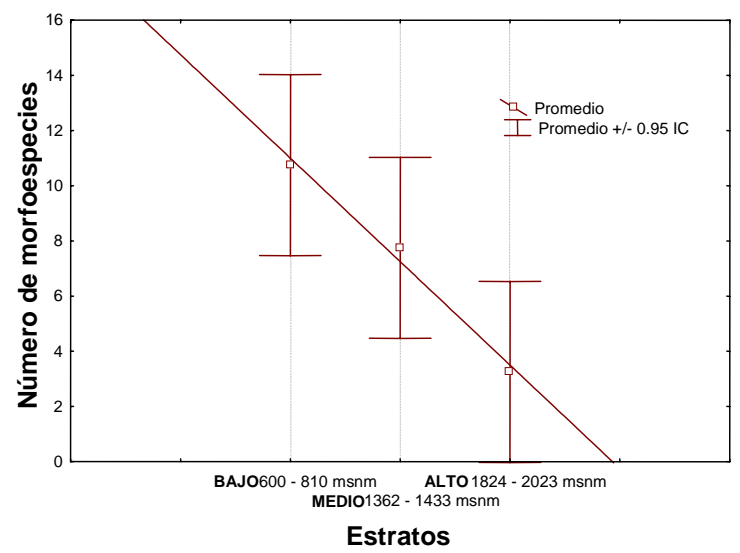

Figura 3. Fluctuación altitudinal de la riqueza de morfoespecies a través de los estratos altitudinales.

A nivel local, analizando la riqueza de especies en el gradiente altitudinal de cada montaña evaluada, el análisis de varianza de dos vías no mostró diferencias significativas entre la riqueza de especies del piedemonte y la cima de la montaña, sin embargo, la Figura 4 muestra que a excepción de la montaña "Alto 2", la riqueza de especies fue más alta en el piedemonte que en la cima de la misma. No se pudo hacer un muestreo en la zona media debido a su elevada pendiente y su difícil acceso. Esta excepción, en donde la riqueza es mayor en la cima que en el piedemonte puede ser debido a que esta última zona se encontraba altamente perturbada por el pastoreo de vacas. Esta perturbación se evidenciaba por la muy baja cantidad de plantas, suelo compactado y las excretas de vacas (Flanagan \& Angulo, 2003; CastroDelgado, com.pers.) condiciones que pudieron haber evitado el establecimiento de nidos de hormigas. No fue posible utilizar otras zonas como opciones.

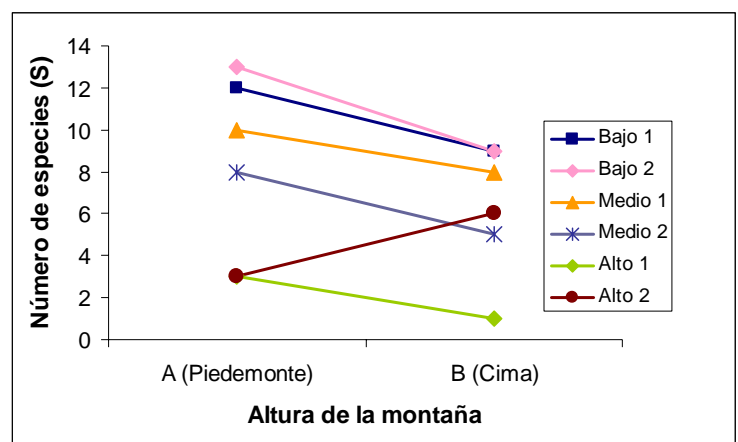

Figura 4 Fluctuación altitudinal de la riqueza de morfoespecies en una montaña. La letra "A" representa el piedemonte de la montaña, siempre al lado de una quebrada y la letra "B" la cima de la misma montaña.

Los patrones altitudinales de las hormigas observados en esta gradiente, tanto a nivel regional como a nivel local (en una montaña) son semejantes a los documentados en estudios previos, en donde la riqueza de hormigas es menor en las zonas altas que en las bajas y medias. Algunos ejemplos, Brühl et al. (1999), quienes estudiaron las comunidades de hormigas de la hojarasca en un gradiente altitudinal en la Montaña Kinabalu en Malasia, con un rango de altitudes desde los 560 hasta los $2600 \mathrm{msnm}$, encontraron que la riqueza de especies es mayor a bajas altitudes y disminuye exponencialmente con el incremento de la altitud. Sin embargo, Samson et al. (1997) encontraron que tanto la riqueza como la abundancia relativa se maximizaron en altitudes medias (en un rango altitudinal de 400 a $800 \mathrm{msnm}$ ) y declinaron abruptamente con el incremento de la altura. Similarmente, Fisher (1998, 1999), estableció que la riqueza y la abundancia se maximizaron en un rango altitudinal de 400 a $800 \mathrm{msnm}$, rango altitudinales medios en sus estudios. Olson (1994), estudió la distribución de los invertebrados de la hojarasca a lo largo de un gradiente altitudinal neotropical y encontró que la gran mayoría de los grupos mostraba máximos de riqueza a altitudes medias. Observó lo mismo para la familia Formicidae, cuyo número de especies se maximizó entre los 750 y $800 \mathrm{msnm}$. Un caso particular ha sido el estudio de Sanders et al. (2003), realizado en las montañas Spring, Nevada (USA), en donde la riqueza de especies de hormigas aumenta con el incremento de la 
altura. Los picos máximos de riqueza de especies han sido relacionados principalmente a factores climáticos, como a la temperatura y la humedad (Grytnes \& McCain, 2007).

Como se puede observar en estudios previos, si la riqueza disminuye de forma lineal o muestra picos máximos a alturas medias parece depender de la escala que se este estudiando. Rahbek (1995, 2005), después de hacer una revisión preliminar sobre estudios de diversidad de diferentes taxa en gradientes altitudinales, encontró que el patrón más común es aquel que muestra los máximos de riqueza a altitudes medias. De la misma manera, Rahbek (2005), resalta que la distribución relativa de los diferentes tipos de patrones varía con la longitud de la gradiente investigada. Así, el porcentaje de estudios donde la máxima riqueza se presenta a alturas medias incremento casi al 70\% después de excluir los estudios que no consideran el gradiente como un todo, es decir, si no incluyen puntos de muestreo desde $\leq 500 \mathrm{a} \geq$ $2000 \mathrm{msnm}$. De esta forma, demostró la importancia de la escala al comprobar que al incluir toda la gradiente los patrones se hacen más marcados. Sin embargo, al comparar los estudios en la escala local (cuando un sólo transecto fue estudiado) con los realizados a escala regional (todo un rango de montañas) observó que al incluir toda la gradiente, el mayor porcentaje de estudios en la escala local son aquellos que muestran los picos máximos de riqueza a alturas medias mientras que en la escala regional este patrón desaparece. El gradiente altitudinal comprendido en este estudio no incluyó alturas más bajas que los $600 \mathrm{msnm}$ debido a limitaciones logísticas, por lo que más estudios futuros que consideren toda la gradiente, es decir, puntos de muestreo desde los $0 \mathrm{msnm}$ hasta los $2600 \mathrm{msnm}$ podrían demostrar si existe un pico máximo de riqueza de especies a alturas medias o efectivamente, una monótona disminución de ésta con el aumento de la altura.

$\underline{\text { Riqueza, Temperatura y Humedad }}$

La temperatura y la humedad relativa, y el efecto combinado entre estos factores, podrían explicar las fluctuaciones de la riqueza de especies tanto a nivel local como a nivel regional, ya que ambas variaron a lo largo de la gradiente. Los valores promedios de la temperatura y humedad y sus respectivos intervalos de confianza tomados en cada una de las estaciones se muestran en la Tabla 3. A nivel regional, la temperatura disminuyó con la altitud $\left(\mathrm{r}^{2}=0.8224 ; \mathrm{r}=\right.$ $-0.9069 ; \mathrm{p}=0.00005 ; \mathrm{y}=34.6611-0.0074 * \mathrm{x})$ y la humedad relativa aumentó con el incremento de la altitud $\left(\mathrm{r}^{2}=0.8354 ; \mathrm{r}=0.9140 ; \mathrm{p}=0.00003 ; \mathrm{y}=\right.$ $47.7518+0.0244 * x)$. Similarmente, la riqueza de morfoespecies aumentó con la temperatura (Figura 9: $r^{2}=0.7514 ; r=0.8668 ; p=0.0003 ; y=-11.8171+$ $0.7675^{*} \mathrm{x}$ ) y disminuyó con la humedad (Figura 10: $\mathrm{r}^{2}$ $=0.8013 ; \mathrm{r}=-0.8952 ; \mathrm{p}=0.00008 ; \mathrm{y}=26.692-$
$0.2429 * \mathrm{x})$. La humedad presente en forma de neblinas, la cual puede estar al menos la mayor parte del año en el estrato alto o en el "Matorral o Sural", reduce la radiación y puede saturar el suelo de agua. La humedad y su efecto combinado con las bajas temperaturas podrían limitar la presencia de especies de hormigas, y en general, de ciertos insectos. Otros estudios también han encontrado asociación entre la temperatura, la humedad y la riqueza de especies de hormigas. Brühl et al. (1999), mencionan una relación similar entre la riqueza de hormigas, la temperatura y la humedad. Sanders et al. (2007) en una investigación dirigida a entender los factores que manejan la diversidad en gradientes altitudinales determinaron que la temperatura limita la diversidad de hormigas a lo largo de estas gradientes. En efecto, establecieron que en los sitios más calientes hay más especies que en los sitios más fríos. La temperatura y la humedad son factores abióticos que regulan las poblaciones de hormigas (Kaspari, 2003; Andersen, 2000; Holldobler \& Wilson, 1990). Las hormigas son termofílicas, y aunque vivan en hábitats muy fríos, son más frecuentes ante la luz del sol. En general, en zonas donde la mayor parte del tiempo el clima es cálido, las hormigas son una parte conspicua del paisaje. Más aún, a excepción de algunas especies tolerantes a las bajas temperaturas, las hormigas soportan difícilmente temperaturas inferiores a los $20{ }^{\circ} \mathrm{C}$ (Holldobler \& Wilson, 1990; Kaspari, 2003).

Utilizando los valores promedios de temperatura, humedad $y$ sus intervalos de confianza y acomodándolos de tal forma que nos permita comparar estas variables entre el piedemonte y la cima de la montaña se puede observar que en muchas de las montañas no hay diferencias significativas entre estas dos zonas (Figura 11 y 12). Las montañas que muestran diferencias son B 2 y M 2 en humedad y en B 2, A 1 y A 2 en temperatura. Aunque la mayoría no muestra diferencias, se observa que en general, tanto para la temperatura como para la humedad, los intervalos de confianza son más cortos en la cima de la montaña que en el piedemonte. La influencia de la temperatura a nivel local sobre la riqueza de hormigas no es tan evidente, sin embargo se puede observar que en las montañas cuya cima estuvo ampliamente separada del piedemonte (como las montañas en el estrato Alto), la temperatura muestra diferencias significativas. En cuanto a la humedad se puede observar que la variación es mucho menor en las cimas de las montañas que en los piedemontes. Esto se debió probablemente a la neblina que circuló por las cimas de las montañas más altas y a la velocidad del viento que tiende a bajar la temperatura. Otros factores como la cobertura vegetal que fue mucho mayor en las zonas bajas pudieron haber influido en estos resultados amortiguando el viento y conservando el calor. 
Composición taxonómica

En general, las subfamilias Formicinae y Myrmicinae fueron las más comunes en los tres estratos, siendo ésta ultima la que tuvo más géneros (42\% del total) y morfoespecies $(56 \%$ del total). Crematogaster, Pheidole (Myrmicinae) y Camponotus (Formicinae) fueron los géneros con más morfoespecies. Aunque el porcentaje de especies de la subfamilia Ponerinae fue bajo, estuvo presente en todos los tipos de bosque. La única subfamilia que se presentó en el estrato bajo fue Dolichoderinae, cuya especie, Tapinoma melanocephalum, parece ser muy conspicua en los bosques secos más bajos del departamento de Lambayeque (Castro-Delgado, com. pers.). En general, esta especie neotropical es muy común en viviendas urbanas y rurales, donde constituye una plaga asociada a ambientes antropogénicos (Cuezzo, 2003). Ésta ha sido ampliamente esparcida por el comercio a través de las regiones tropicales y del mundo (Fernández, 2003).

\section{Diversidad beta}

La Figura 5 muestra el dendrograma construido con los índices de similitud de Jaccard calculados entre las estaciones de muestreo. Tal como se esperaba, los valores de similitud fueron más altos $(45 \%-31 \%)$ entre las estaciones de muestreo de un mismo estrato que entre las estaciones de estratos diferentes. En particular, las estaciones del estrato Bajo que pertenecen a una misma montaña formaron grupos muy definidos, lo que demuestra que estas estaciones comparten más especies entre si que con la otra montaña. Las estaciones "Alto (A) - 2" y "Alto (B) -2 " fueron excepciones ya que se separaron de todo el grupo. Probablemente, debido a las pocas morfoespecies registradas y a que en estas estaciones se colectaron morfoespecies que no fueron encontradas en los otros lugares (Megalomyrmex sp. 1

y Rogeria sp. 2). A nivel regional, al comparar la comunidad de hormigas a través de la gradiente se distingue a cada tipo de hábitat o tipo de bosque con un ensamblaje de especies particular. El grupo conformado por las estaciones de los estratos Bajo y Medio muestra que la composición de morfoespecies de hormigas se presentó más similar entre estos que entre estos y el alto.

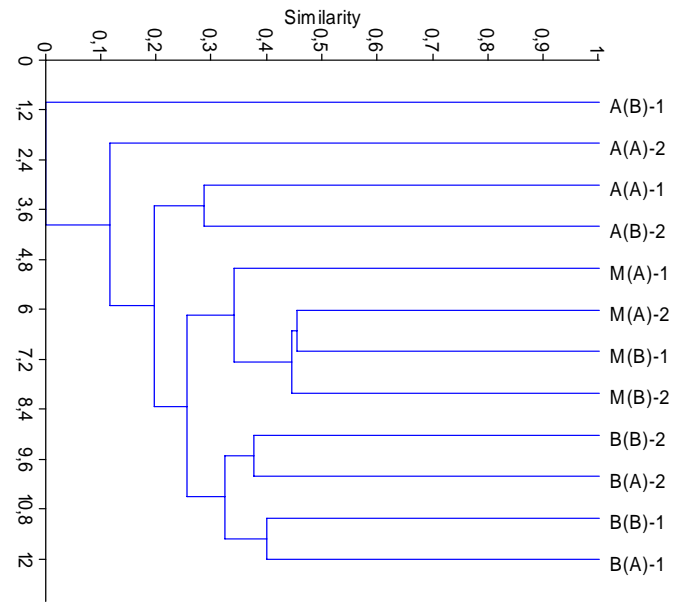

Figura 5. Dendrograma construido con los índices de similitud de Jaccard calculados entre las estaciones de muestreo.

\section{Grupos funcionales}

Tanto en abundancia como en riqueza de especies, los omnívoros superaron a los demás grupos funcionales en todos los estratos altitudinales (Figuras 6 y 7). Los depredadores presentaron un alto número de especies, pero sólo en el estrato alto se mostraron más abundantes. $\mathrm{Su}$ alta abundancia puede relacionarse a los Collembola, cuya abundancia fue mucho mayor en el estrato alto que en el medio y el bajo (datos obtenidos de las muestras, sin publicar). Los collembolas, son comunes y abundantes en el suelo, y necesitan una alta humedad relativa para poder desarrollarse (Borror et al., 1992). Las hormigas depredadoras pueden ejercer una fuerte presión sobre los colémbolos del suelo debido al comportamiento agregado de estos. Las cortadoras de hojas sólo se presentaron en los dos primeros estratos.

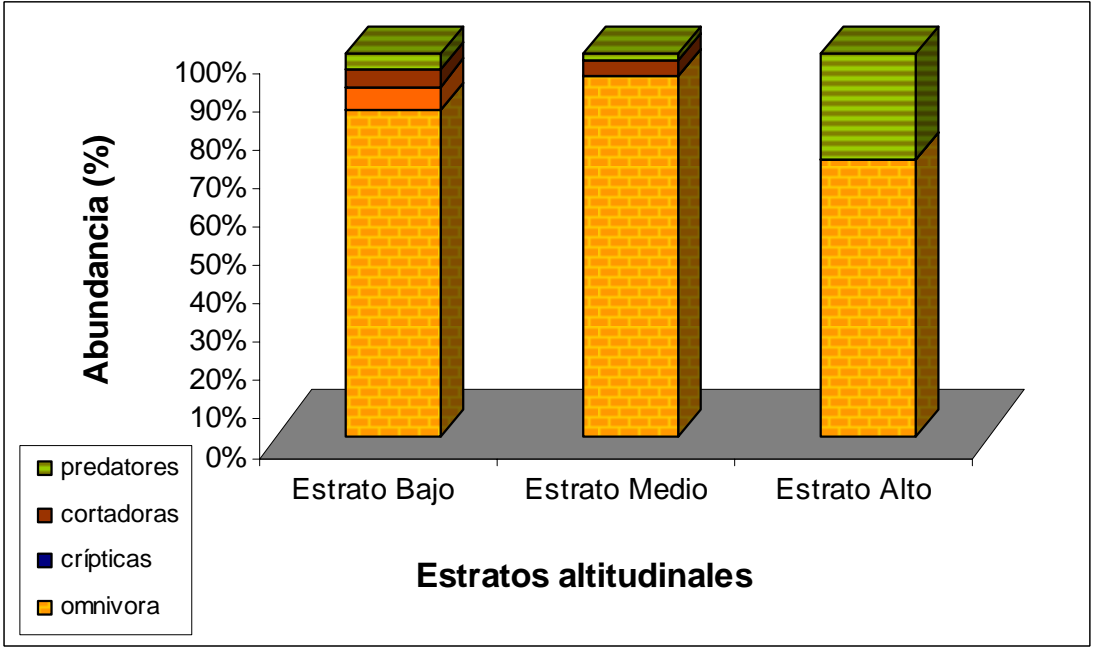

Figura 6. Distribución de los porcentajes de abundancias por categorías tróficas a través de los tres estratos. 


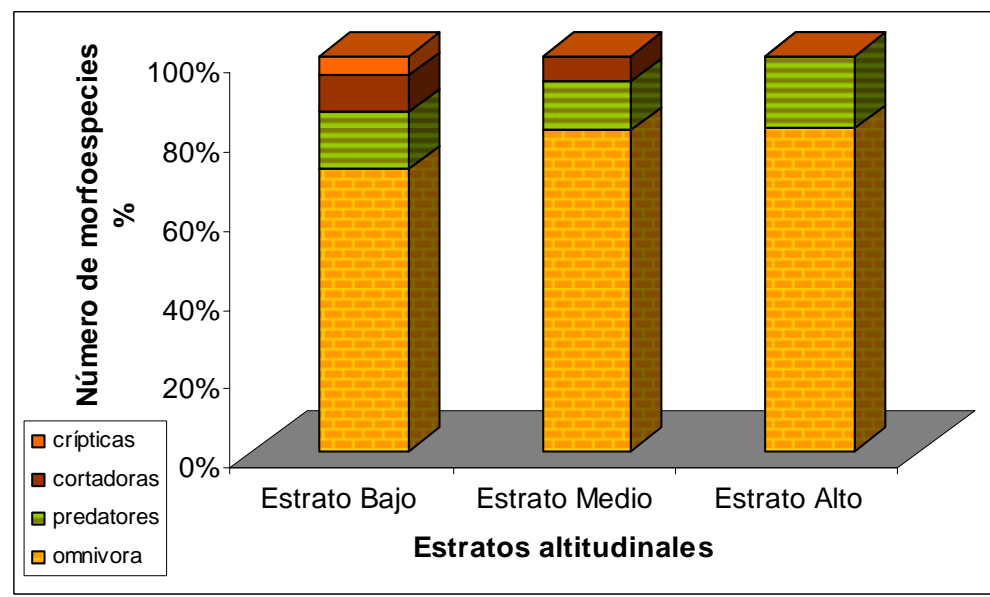

Figura 7. Distribución de los porcentajes del número de morfoespecies por categorías tróficas a través de los tres estratos.

Distribución de cada especie

Este estudio registró una comunidad de hormigas relativamente característica en cada estrato altitudinal ya que sólo una pequeña fracción de la comunidad fue observada en la mayoría de las estaciones. Los tres estratos tuvieron en común sólo 4 especies de hormigas: Camponotus sp. 2, Pheidole sp. 1, Pheidole sp. 2 y Solenopsis sp. 1. La presencia de estas especies en todo el rango altitudinal estudiado sugiere tolerancia a amplios rangos climáticos y geográficos. De las 34 especies registradas con trampas pitfall, 11 especies fueron exclusivas del estrato Bajo, 6 del estrato Medio y 6 del estrato Alto (Tabla 2). Este hecho se puede atribuir a varios factores, entre los cuales se encuentran la complejidad estructural y las condiciones microclimáticas registradas. En el estrato Bajo, la especie más común fue Solenopsis sp. 1 ya que se observó en 21 de las 32 trampas (66\% de las trampas), seguida de Brachymyrmex sp. 1 (31\% de las trampas) y Camponotus sp. 1 (25\% de las trampas). En el estrato Medio, las especies más comunes fueron Pheidole sp. 1, Pheidole sp. $2 \mathrm{y}$ Brachymyrmex sp. 1 ya que se presentaron en el 56; 50 y 47 $\%$ de las 32 trampas, respectivamente. En el estrato Alto Pheidole sp. 1, Pheidole sp. 2 y Solenopsis sp. 1 fueron las más comunes $(13,13$ y $9 \%$ de las 32 trampas, respectivamente). Las especies que fueron más abundantes trampa. tuvieron distribuciones altitudinales más amplias que las menos abundantes (una excepción fue Labidus sp. 1, que cayó únicamente en una trampa). Estas fueron: Brachymyrmex sp. 1 y Pheidole sp. 2 en el $26 \%$; Pheidole sp. 1 en el $28 \%$ y Solenopsis sp. 1 registrada en el $33 \%$ de las 96 trampas (Figura 8), las tres últimas estuvieron presentes en los tres estratos.

Colecta manual

Se registró un total de 30 morfoespecies, de las cuales 16 fueron encontradas en el estrato bajo, 17 en el estrato medio y 0 en el estrato alto. Muchas de estas ya habían sido colectadas con las trampas pitfall. La colecta manual no fue muy útil para confirmar los patrones de diversidad derivados de los registros obtenidos de las trampas pitfall, ya que el estrato Bajo y Medio presentaron casi el mismo número de morfoespecies y en el Alto no se observó ninguna hormiga. Así, la comparación con el último estrato no fue posible. Es muy probable que esto se haya debido a las bajas temperaturas y a la densa humedad, que llego al $100 \%$ casi todos los días que duró el muestreo. Tomando en cuenta que la época seca y la época de lluvias son estaciones muy marcadas y que esta zona se mantiene siempre verde, no se puede asegurar que no haya especies ya que durante el muestreo las colonias podrían haberse protegido en sus respectivos nidos. Por lo que un muestreo en época seca se considera necesario.

\section{TODOS LOS ESTRATOS}

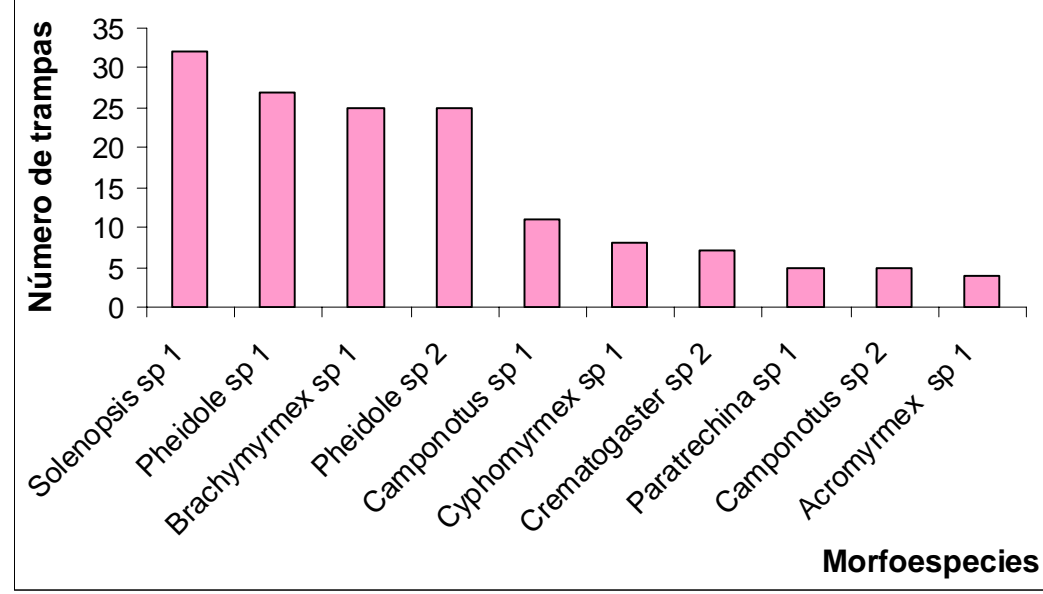

Figura 8. Amplitud de la distribución de las morfoespecies de hormigas registradas en toda la gradiente tomando en cuenta su presencia en cada 


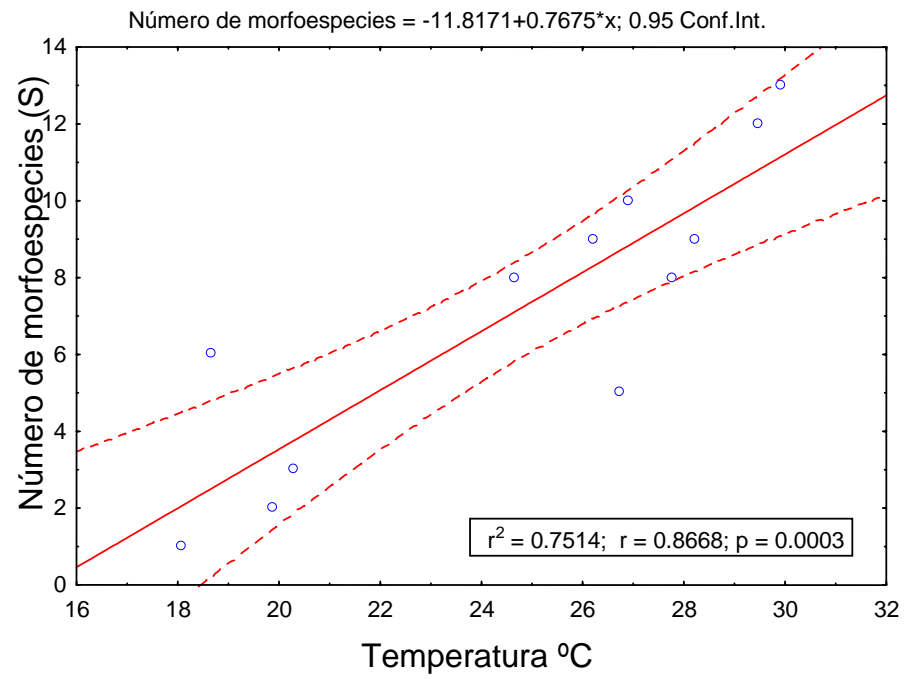

Figura 9. Relación entre la temperatura ${ }^{\circ} \mathrm{C}$ y el número de morfoespecies de hormigas (S) en la gradiente altitudinal muestreada de Laquipampa.

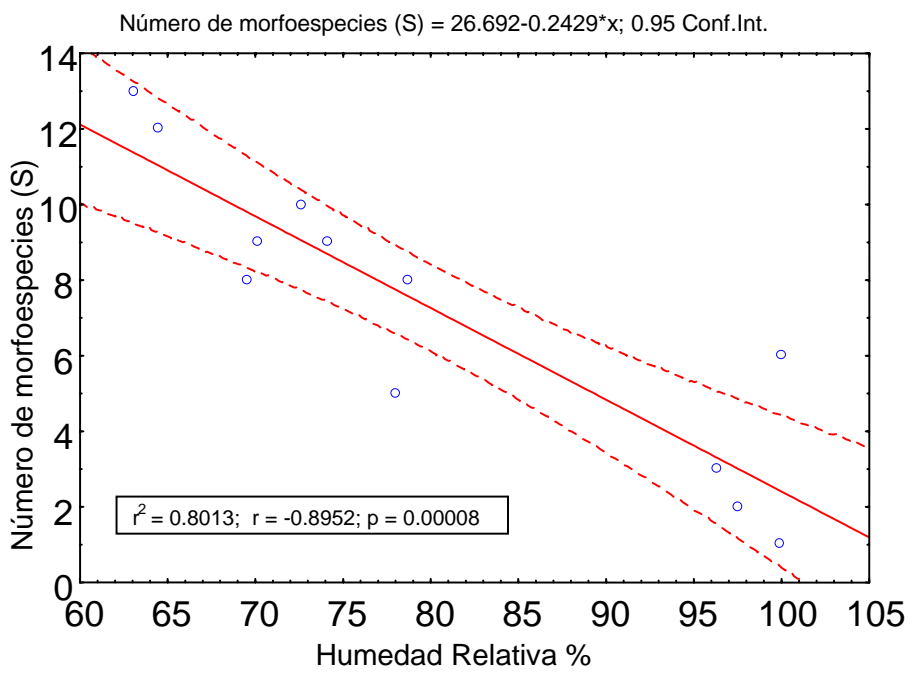

Figura 10. Relación entre la humedad relativa promedio (\%) y el número de especies de hormigas $(\mathrm{S})$ en la gradiente altitudinal muestreada de Laquipampa.

Muchas morfoespecies fueron añadidas a la lista de las colectadas con las trampas pitfall. Así por ejemplo, 8 morfoespecies más fueron registradas exclusivamente para el estrato bajo, como Azteca sp. 1, Azteca sp. 2; Cephalotes sp. 2; Cephalotes sp. 3, Cyphomyrmex sp. 2, Leptothorax sp. 1, Pseudomyrmex sp. 2 y Pseudomyrmex sp. 3. Mientras que 7 especies más fueron registradas sólo para el estrato medio: Labidus sp. 1, Camponotus sp. 4, Crematogaster sp. 4, Crematogaster sp 5, Crematogaster sp 6, Pheidole sp 3 y Ectatomma ruidum. La mayoría de especies registradas con la colecta manual, en especial, las de los géneros Azteca, Cephalotes, Pseudomyrmex y algunas especies del género Crematogaster habitan generalmente en los árboles o en el dosel del bosque
(Holldobler \& Wilson, 1999; Brown, 2000; Andersen, 2000; Longino, 2008), por lo que su presencia en el suelo puede ser eventual.

\section{Conclusiones}

Se registró un total de 892 individuos y 34 especies en 96 trampas pitfall. La colecta manual en transecto registró un total de 30 especies. La combinación de ambos métodos, resultó en 49 especies de hormigas pertenecientes a 21 géneros y 6 subfamilias. Los dos métodos de muestreo generaron dos ensamblajes propiamente diferentes ya que sólo tuvieron 15 especies $(30 \%)$ en común. Los parámetros proporcionados por el ajuste de las curvas a la ecuación de Clench muestran que en general, las trampas pitfall han registrado más del $50 \%$ de las especies de la zona. Sin embargo, se sugiere incrementar el tiempo de muestreo así como el número de trampas. A nivel regional, la riqueza de especies disminuyó con la altura. Esta disminución parece estar relacionada con la disminución de la temperatura y el aumento de la humedad. A nivel local, se determino, en general, que la riqueza es menor en la cima de la montaña que en el piedemonte, lo que podría también estar relacionado con la temperatura y la humedad. La composición de morfoespecies fue muy similar entre las estaciones de muestreo de un mismo estrato de tal forma que estas formaron grupos definidos. $\mathrm{La}$ composición de especies fue más similar entre el estrato Bajo y el Medio que entre estos y el alto. Los tres estratos fueron dominados por omnívoros y tuvieron en común sólo 4 especies de hormigas: Camponotus sp. 2, Pheidole sp. 1, Pheidole sp. 2 y Solenopsis sp. 1. De las 34 especies registradas con trampas pitfall, 11 especies fueron exclusivas del estrato Bajo, 6 del estrato Medio y 6 del estrato Alto. Se espera que estudios futuros en toda la gradiente puedan ayudar a determinar picos máximos de riqueza de especies.

\section{Agradecimientos}

Al ingeniero forestal Fernando Angulo Pratolongo, director de la Asociación Cracidae Perú, por financiar parte de este proyecto. A los asistentes de campo, los señores Oscar Rodríguez Durand y Napoleón Durand de la Cruz por su guía y ayuda en la colocación de las trampas. A Idea Wild por financiar la compra de gran parte de los materiales. Al Dr. Edgar Sánchez por sus consejos al inicio del trabajo; al Blgo. Alfredo Giraldo por su ayuda en el análisis del muestreo; al Dr. Fabrizio Lanza por su apoyo constante e incondicional 


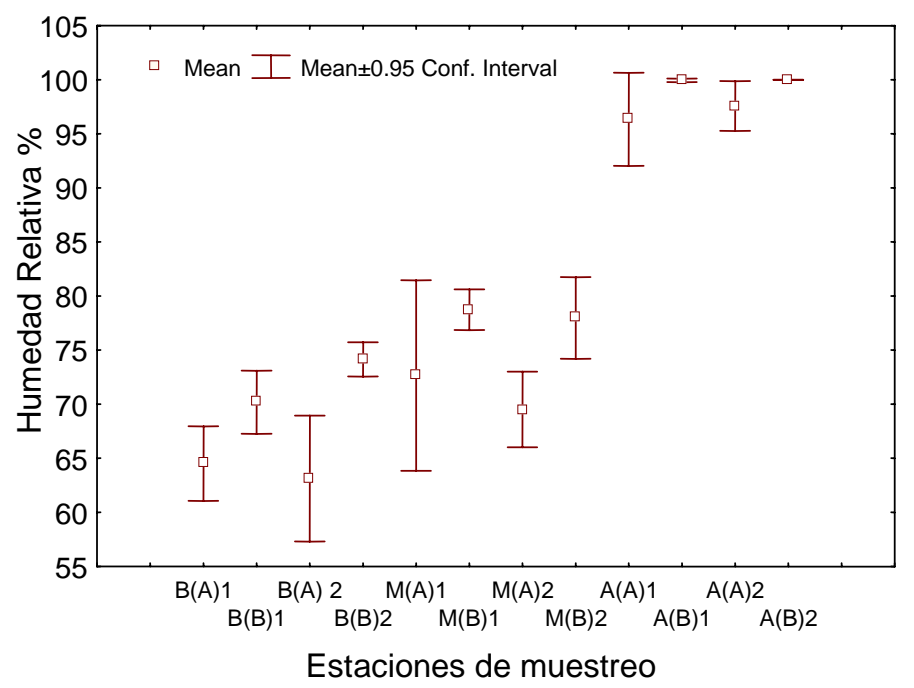

Figura 11. Humedad relativa promedio y sus intervalos de confianza en cada estación de muestreo. $\mathrm{B}(\mathrm{A}) 1$ y $\mathrm{B}(\mathrm{B}) 1$ representan el piedemonte y la cima de una montaña, respectivamente.

y al estudiante de biología Freddy Molina por su ayuda en el conteo de Collembola.

\section{Literatura citada}

Agosti D. \& Johnson N.F. Editors. 2005. Antbase. (En Línea). Disponible en http://www.antbase.org/. Consultado el 28 de Octubre de 2008.

Alonso L.E. \& Agosti D. 2000. Biodiversity Studies, Monitoring and Ants: An Overview. In: D. Agosti J.D. Majer L. Alonso \& T. Shultz (Eds.) Ants, Standard Methods for Measuring and Monitoring Biodiversity. Smithsonian Institution Press. Capítulo 1: 1-8.

Andersen A. 1991. Sampling communities of ground foraging ant: Pitfall catches compared with quadrant count in an Australian tropical savanna. Australian Journal of Ecology. 16: 273 -276.

Andersen A. 1993. Ants as Indicators of Restoration Success at a Uranium Mine in Tropical Australia. Restoration Ecology. 5(2):156 -167.

Andersen A.N. 2000. A global ecology of rain forest ants: functional groups in relation to stress and disturbance. In: D. Agosti J.D. Majer, L. Alonso \& T. Shultz (Eds.) Ants: Standard Methods for Measuring and Monitoring Biodiversity. Smithsonian Institution Press, Washington D.C., USA. Capítulo 3: 25 -34.

Andersen A. N., Hoffmann, B. D. \& Somes J. 2003. Ants as indicators of minesite restoration: community recovery at one of eight rehabilitation sites in central Queensland. Ecological Management Restoration. 4: S12-S19.

Andersen A.N. \& Majer J.D. 2004. Ant show the way Down Under: Invertebrates as bioindicators in land management. Front. Ecol. Environ. 2(6): 291-298

Angulo P.F. \& Alemán de Lama D. (Eds.). 2006. Expedición Laquipampa 2006 - Informe Preliminar, Marzo 2006. Asociación Cracidae Perú, Chiclayo, Perú.

Bolton B. 1994. Identification Guide to the Ant Genera of the World. Harvard University Press, Cambridge, Massachusetts.
Bolton B. 1995. A New General Catalogue of the Ants of the World. Harvard University Press, Cambridge, Massachusetts.

Borror D.J., Triplehorn C.A. \& Johnson N.F. 1992. Study of Insects. 6ta Edición, Saunders College Publishing Harcourt Brace College Publishers.

Brise D.T. 1982. The effect of ants on the soil of a semi-arid saltbush habitat. Insects Sociaux. 29: 375 -382.

Brown W.L.Jr. 2000. Diversity of ants. In: D. Agosti J. D. Majer L. E. Alonso \& T. R. Schultz (Eds.) Ants: Standard Methods for Measuring and Monitoring Biodiversity. Smithsonian Institution Press. Capítulo 5: $45-79$.

Brühl C.A., Mohamed M. \& Linsenmair K.E. 1999. Altitudinal distribution of leaf litter ants along a transect in primary forest on Mount Kinabalu, Sabah, Malaysia. Journal of Tropical Ecology.15: 265-267.

Clarke K.R. \& Warwick R.M. 1996. Changes in Marine Communities. An approach to statistical analysis and interpretation Plymouth Marine Laboratory Plymouth Routines in Multivariate Ecological Research. Plymouth. U.K.

Cuezzo F. 2003. Subfamilia Dolichoderinae. Capitulo 20. En: Fernández F. (Ed.) Introducción a las Hormigas de la región Neotropical. Instituto de Investigación de Recursos Biológicos Alexander von Humboldt, Bogotá, Colombia.

Davidson D.W. 1998. Resource discovery versus resource domination in ants: breaking the trade-off. Ecol. Ent. 23: 484-490.

Davidson D.W. \& Patrell-Kim L. J.1996. Tropical arboreal ants: Why so abundant?.In: Gibson A. (Ed.) Neotropical Biodiversity and Conservation. UCLA Herbarium Publ. 1:127-140. Los Angeles.

Davidson D.W., Cook S.C., Snelling R.R. \& Chua, T.H. 2003. Explaining the abundance of ants in lowland tropical rainforest canopies. Science.300: 969-972.

De Leo G.A. \& Levin S. 1997. The multifaceted aspects of ecosystem integrity. Conservation Ecology. 1(1): 3. Disponible en: http://www.consecol.org/vol1/iss1/art3/ 
Derraik J.G.B., Dickinson K.J.M., Closs G., Sirvid P., Barratt B.I.P. \& Patrick B. 2002. Arthropod morphospecies vs. taxonomic species: a case study with Araneae, Coleoptera and Lepidoptera. Conservation Biology.16: 1015-1023.

Dinerstein E., Olson D.M., Graham D.J., Webster A.L., Primm S.A., Bookbinder M.P. \& G. Ledec. 1995. A conservation assessment of the terrestrial ecoregions of Latin America and the Caribbean. The World Bank, Washington, DC, USA.

Dourojeanni M. 2006. Estudio de caso sobre la carretera interoceánica en la amazonia sur del Perú. Lima, Perú.

Dudley N. \& Stolton S. (Eds). 2003. Running Pure: The Importance of Forest Protected Areas to Urban Water Supply. WWF and the World Bank, Gland, Switzerland and Washington DC, USA.

Fernández F. 2003. Introducción a las Hormigas de la región Neotropical. Instituto de Investigación de Recursos Biológicos Alexander von Humboldt, Bogotá, Colombia.

Fisher B.L. 1998. Ant diversity patterns along an elevational gradient in the Réserve Spéciale d'Anjanaharibe-Sud and on the western Masoala Peninsula, Madagascar. Fieldiana: Zoology (n.s.) 90: 39-67.

Fisher B.L. 1999. Ant diversity patterns along an elevational gradient in the Réserve Naturelle Intégrale d'Andohahela, Madagascar . Fieldiana: Zoology (n.s.) 94: 129-147.

Fittkau E.J. \& Klinge H. 1973. On biomass and trophic structure of the central Amazonian rain forest ecosystem. Biotropica.5(1): 2-14.

Flanagan J.N.M. \& Angulo P.F. 2003. La Zona Reservada de Laquipampa - Ecología, Conservación y Manejo. En: Memorias del Primer Congreso Internacional Bosques Secos (6-9 Nov. 2003). Universidad de Piura, Perú.

Folgarait P.J. 1998. Ant biodiersity and its relationship to ecosystem functioning: a review. Biodiversity and Conservation.7: 1221-1244

Giraldo M.A. 2002. Análisis de los patrones de variación espacio-temporal de las poblaciones de coleópteros en la Reserva Nacional de Lachay durante el periodo 1998 2001. Tesis para optar el Título de Biólogo - UNALM.

Giraldo M.A. \& Arellano C.G. 2003. Resiliencia de la comunidad epígea de Coleoptera en las Lomas de Lachay después del evento El Niño 1997-98. Ecología Aplicada. 2(1): $60-68$.

Grytnes J.A. \& McCain C.M. 2007. Elevational Trends in Biodiversity. Encyclopedia of Biodiversity. 2: 1-8.

Gunadi B. \& Verhoef H.A. 1993. The flow of nutrients in a Pinus merkusii forest plantation in Central Java: the contribution of soil animals. European Journal of Soil Biology. 29: 133-139.

Haines B.L. 1978. Element and energy flows through colonies of the leaf cutting ant, Atta colombica, in Panama. Biotropica. 10(4): 270-277.

Holdridge L.R. 1982. Ecología basada en zonas de vida. IICA, San José, Costa Rica.

Holldobler B. \& Wilson E.O. 1990. The Ants. Belknap Press, Cambridge, Massachusetts.

Jiménez-Valverde A. \& Hortal J. 2003. Las curvas de acumulación de especies y la necesidad de evaluar la calidad de los inventarios biológicos. Revista Ibérica de Aracnología. 8: 151-161.
Kaspari M. 2003. Introducción a la ecología de las hormigas. Capitulo 6. En: Fernández, F. (Ed.) Introducción a las Hormigas de la región Neotropical. Instituto de Investigación de Recursos Biológicos Alexander von Humboldt, Bogotá, Colombia.

Kattan G. 2002. Fragmentación: patrones y mecanismos de extinción de especies. Págs: 561-590 En: Guariguata M., \& Kattan G. (Eds.) Ecología y Conservación de los Bosques Neotropicales. Libro Universitario Regional (EULAG-GTZ)

Kremen C., Colwell R.K., Erwin T.L., Murphy D.D., Noss R.F. \& Sanjayan, M.A. 1993. Terrestrial Arthropod Assemblages: Their Use in Conservation Planning. Conservation Biology. 7(4): 796 - 808.

Kricher J. 2006. Un Compañero Neotropical: Una introducción a los animales, plantas, y ecosistemas del Trópico del Nuevo Mundo. 2da Edicion. American Birding Association. USA.

Longino J.T. 2008. Ants of Costa Rica. Disponible en http://academic.evergreen.edu/projects/ants/. Consultado el 3 de Julio de 2008.

Magurran A.E. 1988. Diversidad Ecológica y su Medición. Ediciones VEDRA. Barcelona. $200 \mathrm{p}$.

Majer J.D. 1983. Ants: Bio - indicators of Minesite Rehabilitation, Land - Use, and Land Conservation. 1983. Environmental Management.7(4): 375-383.

Maravi E., Norgrove L., Amanzo J. \& Sissa A. 2003. Identificación preliminar de prioridades para la conservación del Oso de Anteojos (Tremarctos ornatus) y el Tapir de Montaña (Tapirus pinchaque) en la Subdivisión Perú de la Ecoregión de los Andes del Norte. WWF - Perú.

Margalef R. 1991. Ecología. Ediciones Omega S. A. 7ma edición.

Myers N., Mittermeier R.C., Mittermeier G., Da Fonseca A.B. \& Kent J. 2000. Biodiversity hotspots for conservation priorities. Nature. 403: 853 - 858.

Olson D.M. 1994. The distribution of leaf litter invertebrates along a neotropical altitudinal gradient. Journal of Tropical Ecology. 10: 129 - 150.

Pearson D.L. \& Cassola F. 1992. World - Wide Species Richness Patterns of Tiger Beetles (Coleoptera: Cicindelidae): Indicador Taxon for Biodiversity and Conservation Studies. Conservation Biology. 6(3): 376 391.

Peterson G., Craig R.A. \& Holling C.S. Ecological Resilience, Biodiversity, and Scale. Ecosystems. 1(1): 618.

Rosenberg D.M., Danks H.V. \& Lehmkuhl D.M. 1986. Importance of Insects in Enviromental Impact Assessment. Environmental Management. 10(6):773783.

Rahbek C. 1995. The elevational gradient of species richness: a uniform pattern?. Ecography. 18 (2): 200205.

Rahbek C. 2005.The role of spatial scale and the perception of large - scale species - richness patterns. Ecology Letters. 8: 224-239.

Samson D.A., Rickart E.A. \& Gonzales P.C. 1997. Ant diversity and abundance along an elevational gradient in the Philippines. Biotropica. 29: 349-363.

Sanders N.J. 2002. Elevational gradients in ant distributions: area, species richness, and Rapoport's rule. Ecography. 25: $25-32$. 
Sanders N.J., Moss J. \& Wagner D. 2003. Patterns of ant species richness along elevational gradients in an arid ecosystem. Global Ecology and Biogeography. 12: 93102.

Sanders N.J., Lessard J.P., Dunn R.R. \& Fitzpatrick M.C. 2007. Temperature, but not productivity or geometry, predicts elevational diversity gradients in ants across spatial grains. Global Ecology and Biogeography. 16(5):640-649.

Silvestre R. Brandão C.R.F. \& Silva R.R. 2003. Grupos funcionales de hormigas: el caso de los gremios del Cerrado. En: F. Fernández (Ed.) Introducción a las hormigas de la Región Neotropical. Smithsonian Institution Press.

StatSoft Inc. 2004. STATISTICA (data analysis software system), version 7. www.statsoft.com.

Steel R. \& Torrie J. 1988. Bioestadística: principios y procedimientos. Mc Graw-Hill Interamericana de Mexico.

Stevens G.C. 1992. The elevational gradient in altitudinal range: an extension of Rapoport's latitudinal rule to altitude. American Naturalist. 140: 893-911.
Terborgh J. 1977. Bird species diversity on an Andean elevational gradient. Ecology. 58: 1007-1019.

Verhaagh, M. 1990. The Formicidae of the rain forest in Panguana, Peru: the most diverse local ant fauna ever recorded. Págs 217-218. En: G.K. Veeresh, B. Mallick \& C.A. Viraktamath, (Eds). Social insects and the environment. Oxford and IBH Publishing, New Delhi

Verhaagh, M. 1991. Clearing a tropical rain forest effects on the ant fauna. Págs 59- 68. In: W. Erdelen, N. Ishwaran \& P. Miiller (Eds). Proceedings of the International and Interdisciplinary Symposium on Tropical Ecosystems. Margraf Scientific, Weikersheim, Germany

Wheeler W.M. 1965. ANTS, Their Structure, Development and Behavior. Columbia University Press New York and London.

Whitmore T.C. 1997. Tropical Forest Disturbance, Disappearance and Species Loss. En: Laurance R.W. \& Bierregaard R.O. (Eds). Tropical Forests Remnants: Ecology, Management and Conservation of Fragmented Communities. The University of Chicago Press.

Wilson E.O. 1987. The Arboreal Ant fauna of Peruvian Amazon Forest: A First Assessment. Biotropica. 19 (3): 245-251.

\section{Tablas citadas en el texto}

Tabla 1. Coeficientes de determinación (R2) de las curvas y parámetros obtenidos por el ajuste de las curvas a la ecuación de Clench para seis estaciones de muestreo. Sobs/(a/b) muestra la proporción de fauna registrada en cada estación evaluada, n 0.95 y n 0.70 , muestran cuantas trampas pitfall (con un esfuerzo temporal de 84 horas) se necesitarían para obtener el $95 \%$ y el $70 \%$ de las especies

\begin{tabular}{lcccccc}
\hline & $\begin{array}{c}\text { Baja } \\
\text { A-1 }\end{array}$ & $\begin{array}{c}\text { Baja } \\
\text { A-2 }\end{array}$ & $\begin{array}{c}\text { Media } \\
\text { A-1 }\end{array}$ & $\begin{array}{c}\text { Media } \\
\text { A-2 }\end{array}$ & $\begin{array}{c}\text { Alta } \\
\text { A-1 }\end{array}$ & $\begin{array}{c}\text { Alta } \\
\text { B-2 }\end{array}$ \\
\hline \hline R2 & 0.9985 & 0.9984 & 0.9997 & 0.9985 & 0.9963 & 0.9993 \\
Sobs/(a/b)*100 & 64 & 50 & 62 & 61 & 58 & 50 \\
Núm. Especies & & & & & & \\
estimadas & 24 & 22 & 16 & 15 & 5 & 12 \\
n 0.95 & 155 & 88 & 94 & 97 & 104 & 137 \\
n $\mathbf{0 . 7 0}$ & 19 & 11 & 12 & 12 & 13 & 17
\end{tabular}




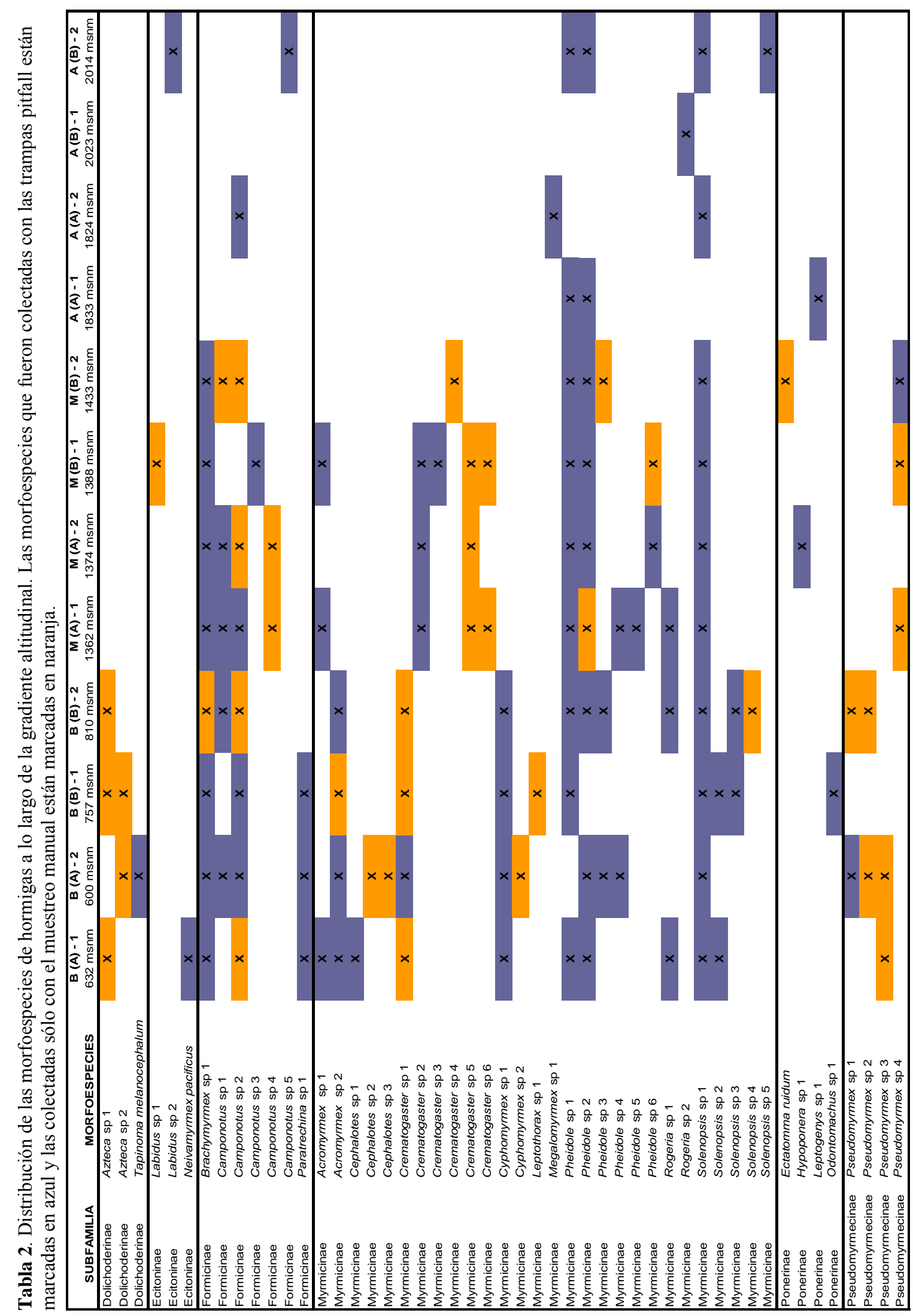


Tabla 3. Temperatura $\left({ }^{\circ} \mathrm{C}\right)$ y humedad relativa promedio $(\%)$ con sus respectivos intervalos de confianza y la riqueza de morfoespecies de hormigas en cada estación de muestreo.

\begin{tabular}{ccccccccc}
\hline Estación & msnm & \multicolumn{3}{c}{ TEMPERATURA } & \multicolumn{2}{c}{ HUMEDAD } & RIQUEZA \\
\hline $\mathrm{B}(\mathrm{A}) 1$ & 632 & 29.47 & \pm & 0.89 & 64.52 & \pm & 2.70 & 12 \\
$\mathrm{~B}(\mathrm{~A}) 2$ & 600 & 29.93 & \pm & 1.45 & 63.13 & \pm & 4.56 & 13 \\
$\mathrm{~B}(\mathrm{~B}) 1$ & 757 & 28.23 & \pm & 0.66 & 70.18 & \pm & 2.29 & 9 \\
$\mathrm{~B}(\mathrm{~B}) 2$ & 810 & 26.20 & \pm & 0.39 & 74.15 & \pm & 1.25 & 9 \\
\hline $\mathrm{M}(\mathrm{A}) 1$ & 1362 & 26.90 & \pm & 1.69 & 72.65 & \pm & 6.90 & 10 \\
$\mathrm{M}(\mathrm{A}) 2$ & 1374 & 27.77 & \pm & 2.01 & 69.52 & \pm & 2.74 & 8 \\
$\mathrm{M}(\mathrm{B}) 1$ & 1388 & 24.67 & \pm & 0.18 & 78.75 & \pm & 1.47 & 8 \\
$\mathrm{M}(\mathrm{B}) 2$ & 1433 & 26.75 & \pm & 1.05 & 77.98 & \pm & 2.96 & 5 \\
\hline $\mathrm{A}(\mathrm{A}) 1$ & 1833 & 20.30 & \pm & 0.66 & 96.35 & \pm & 3.37 & 3 \\
$\mathrm{~A}(\mathrm{~A}) 2$ & 1824 & 19.88 & \pm & 0.16 & 97.58 & \pm & 1.80 & 3 \\
$\mathrm{~A}(\mathrm{~B}) 1$ & 2023 & 18.07 & \pm & 0.37 & 99.93 & \pm & 0.13 & $\mathbf{1}$ \\
$\mathrm{A}(\mathrm{B}) 2$ & 2014 & 18.67 & \pm & 0.40 & 100.0 & \pm & 0.00 & 6 \\
\hline
\end{tabular}

\footnotetext{
${ }^{1}$ Museo de Entomología “Klaus Raven Büller” y Laboratorio de Ecología de Insectos del Departamento de Biología. Universidad Nacional Agraria La Molina. Av. La Molina s/n, Apartado postal 12-056. Lima 12 - Perú. Correo: silviacastro@lamolina.edu.pe

${ }^{2}$ Departamento de Estadística. Universidad del Estado de Carolina del Norte, Estados Unidos de América. P.O. Box 8203. NC 27695, EE.UU.
} 